\title{
The role of $\mathrm{m}^{6} \mathrm{~A}$ RNA methylation in human cancer
}

\author{
Xiao-Yu Chen, Jing Zhang ${ }^{*}$ and Jin-Shui Zhu ${ }^{*}$ (D
}

\begin{abstract}
$\mathrm{N}^{6}$-methyladenosine $\left(\mathrm{m}^{6} \mathrm{~A}\right)$ is identified as the most common, abundant and conserved internal transcriptional modification, especially within eukaryotic messenger RNAs (mRNAs). $\mathrm{M}^{6} \mathrm{~A}$ modification is installed by the $\mathrm{m}^{6} \mathrm{~A}$ methyltransferases (METTL3/14, WTAP, RBM15/15B and KIAA1429, termed as "writers"), reverted by the demethylases (FTO and ALKBH5, termed as "erasers") and recognized by $\mathrm{m}^{6} \mathrm{~A}$ binding proteins (YTHDF1/2/3, IGF2BP1 and HNRNPA2B1, termed as "readers"). Acumulating evidence shows that, $m^{6} A$ RNA methylation has an outsize effect on RNA production/metabolism and participates in the pathogenesis of multiple diseases including cancers. Until now, the molecular mechanisms underlying $m^{6} \mathrm{~A}$ RNA methylation in various tumors have not been comprehensively clarified. In this review, we mainly summarize the recent advances in biological function of $\mathrm{m}^{6} \mathrm{~A}$ modifications in human cancer and discuss the potential therapeutic strategies.
\end{abstract}

Keywords: N6-methyladenosine, Cancer, RNA methylation, Prognosis, Growth, Metastasis

\section{Introduction}

According to MODOMICS, 163 different chemical modifications in RNA have been identified in all living organisms by the end of 2017 [1]. Among these modifications, $\mathrm{N}^{6}$-methyladenosine $\left(\mathrm{m}^{6} \mathrm{~A}\right)$, methylated at the $\mathrm{N}^{6}$ position of adenosine, has been considered as the most pervasive, abundant and conserved internal transcriptional modification within eukaryotic messenger RNAs (mRNAs) [2], microRNAs (miRNAs) [3] and long non-coding RNAs (lncRNAs) [4]. RNA $\mathrm{m}^{6} \mathrm{~A}$ is enriched near stop codon and $3^{\prime}$ untranslated terminal region (UTR) $[5,6]$ and translated near $5^{\prime}$ UTR in a capindependent manner [7], thereby affecting RNA transcription, processing, translation and metabolism.

The deposition of $\mathrm{m}^{6} \mathrm{~A}$ is encoded by a methyltransferase complex involving three homologous factors jargonized as 'writers', 'erasers' and 'readers' (Fig. 1). Methyltransferaselike 3 (METTL3) [8], METTL14 [9], Wilms tumor 1associated protein (WTAP) [10], RBM15/15B [11] and KIAA1429 [12] are categorized as the components of 'writers' that catalyze the formation of $\mathrm{m}^{6} \mathrm{~A}$; 'erasers', fat mass and obesity-associated protein (FTO) [13] and alkB homologue 5 (ALKBH5) [14], selectively remove the

\footnotetext{
*Correspondence: jing5522724@vip.163.com; zhujs1803@163.com Department of Gastroenterology, Shanghai Jiao Tong University Affiliated Sixth People's Hospital, Yishan Road 600, Shanghai 200233, China
}

methyl code from target mRNAs; 'Readers' are capable of decoding $\mathrm{m}^{6} \mathrm{~A}$ methylation and generating a functional signal, including YT521-B homology (YTH) domaincontaining protein [15], eukaryotic initiation factor (eIF) 3 [11], IGF2 mRNA binding proteins (IGF2BP) families [16] and heterogeneous nuclear ribonucleoprotein (HNRNP) protein families [17]. YTH domain can recognize $\mathrm{m}^{6} \mathrm{~A}$ through a conserved aromatic cage [18] and another two proteins FMR1, LRPPRC "read" this modification $[19,20]$. Contrary to the conventional 'writer'-'eraser'-'reader' paradigm, few studies reveal METTL3/16 as a $\mathrm{m}^{6} \mathrm{~A}$ 'writer' or 'reader' [21].

$\mathrm{M}^{6} \mathrm{~A}$ RNA modification is a dynamic and reversible process which was corroborated by the discovery of 'eraser' in 2011 [13]. It is associated with multiple diseases such as obesity, infertility and cancer [22]. In this review, we summarize the function and therapeutic advances of $\mathrm{m}^{6} \mathrm{~A}$ modifications in human cancer and provide their promising applications in the treatment of these malignant tumors (Table 1).

\section{Biological function of $\mathrm{m}^{6} \mathrm{~A}$ modification in mammals}

Recent years have witnessed a substantial progress of $\mathrm{m}^{6} \mathrm{~A}$ post-transcriptional modification in regulating RNA transcription [23, 24], processing event [25-27], 


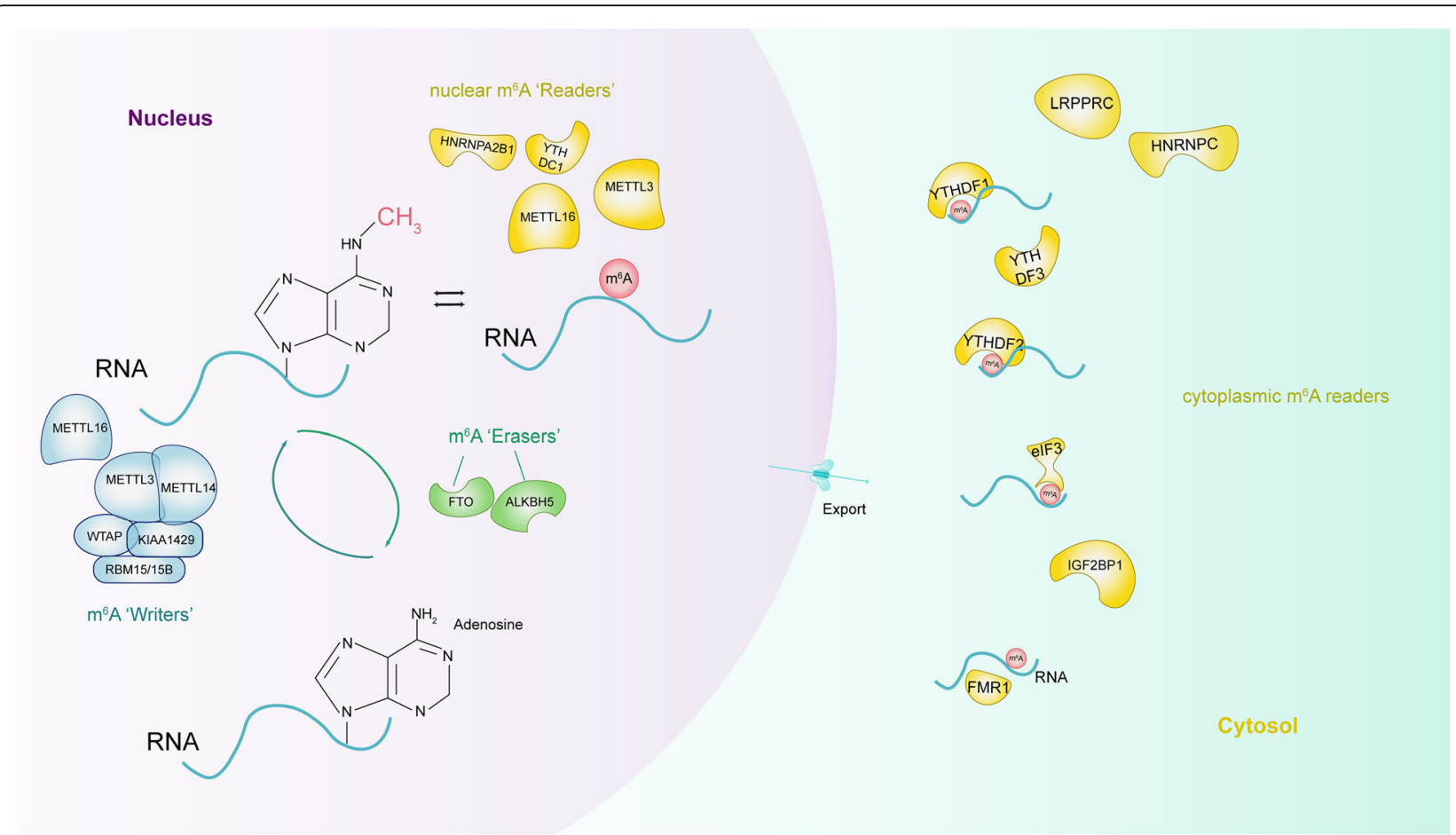

Fig. 1 Molecular composition of $\mathrm{m}^{6} \mathrm{~A}$ RNA methylation. $\mathrm{M}^{6} \mathrm{~A}$ methylation is a dynamic and reversible process coordinated by a series of methyltransferases (METTL3/14, WTAP, RBM15/15B, and KIAA1429, termed as " $\mathrm{m}^{6} \mathrm{~A}$ writers"), demethylases (FTO and ALKBH5, " $m{ }^{6} \mathrm{~A}$ erasers") and identifiers (YTHDF1/2/3, YTHDC1, HNRNPA2B1, HNRNPC, elF3, FMR1, and LRPPRC, " $m$ A. 'Readers")

splicing [28-33], RNA stabilities [34-40] and translation [42-49] (Fig. 2).

\section{$M^{6} A$ modification in RNA transcript}

METTL3 and FTO are implicated in regulating transcription of CCAAT-enhancer binding protein (CEBP) family. METTL3 is localized to the starting sites of CEBPZ, which is required for recruitment of METTL3 to chromatin [23]. CEBPA is identified as an exclusive transcription factor displaying a positive correlation with FTO and regulating its transcription in acute myeloid leukemia (AML) [24].

\section{$M^{6} \mathrm{~A}$ modification in RNA processing}

$\mathrm{M}^{6} \mathrm{~A}$ modifications promote the initiation of miRNA biogenesis [3] and regulate nuclear mRNA processing events [25]. METTL3 recognizes the pri-miRNAs by microprocessor protein DGCR8 and causes the elevation of mature miRNAs and concomitant reduction of unprocessed pri-miRNAs in breast cancer [3]. METTL14 interacts with DGCR8 to modulate pri-miR-126 and suppresses the metastatic potential of hepatocellular carcinoma (HCC) [26]. FTO can regulate poly(A) site and 3' UTR length by interacting with METTL3 [25]. YTHDC1 knockout in oocytes exhibits massive defects and contributes to extensive alternative polyadenylation and 3' UTR length alterations [27].

\section{$M^{6} A$ modification in RNA splicing}

$M^{6}$ A RNA modifications that overlap in space with the splicing enhancer regions affect alternative RNA splicing by acting as key pre-mRNA splicing regulators [28]. Inhibiton of $\mathrm{m}^{6} \mathrm{~A}$ methyltransferase impacts gene expression and alternative splicing patterns [29]. FTO regulates nuclear mRNA alternative splicing by binding with SRSF2 [25]. FTO and ALKBH5 regulate $\mathrm{m}^{6} \mathrm{~A}$ around splice sites to control the splicing of Runtrelated transcription factor 1 (RUNX1T1) in exon [28], and removal of $\mathrm{m}^{6} \mathrm{~A}$ by FTO reduces the recruitment of SRSF2 and prompts the skipping of exon 6, leading to a short isoform of RUNX1T1 [30]. Depletion of METTL3 is associated with RNA splicing in pancreatic cancer [31]. WTAP is enriched in some proteins involved in pre-mRNA splicing [32]. But, some studies show that, $\mathrm{M}^{6} \mathrm{~A}$ is not enriched at the ends of alternatively spliced exons and METTL3 unaffects pre-mRNA splicing in embryonic stem cells [33].

\section{$M^{6} A$ modification in RNA degradation}

$\mathrm{M}^{6} \mathrm{~A}$ is a determinant of cytoplasmic mRNA stability [34], and reduces mRNA stability [35]. A RNA decay monitoring system is adopted to investigate the effects of $\mathrm{m}^{6} \mathrm{~A}$ modifications on RNA degradation [36]. Knockdown of METTL3 abolishes SOCS2 $\mathrm{m}^{6} \mathrm{~A}$ modification and augments SOCS2 expression [37]. $\mathrm{M}^{6} \mathrm{~A}$-mediated 


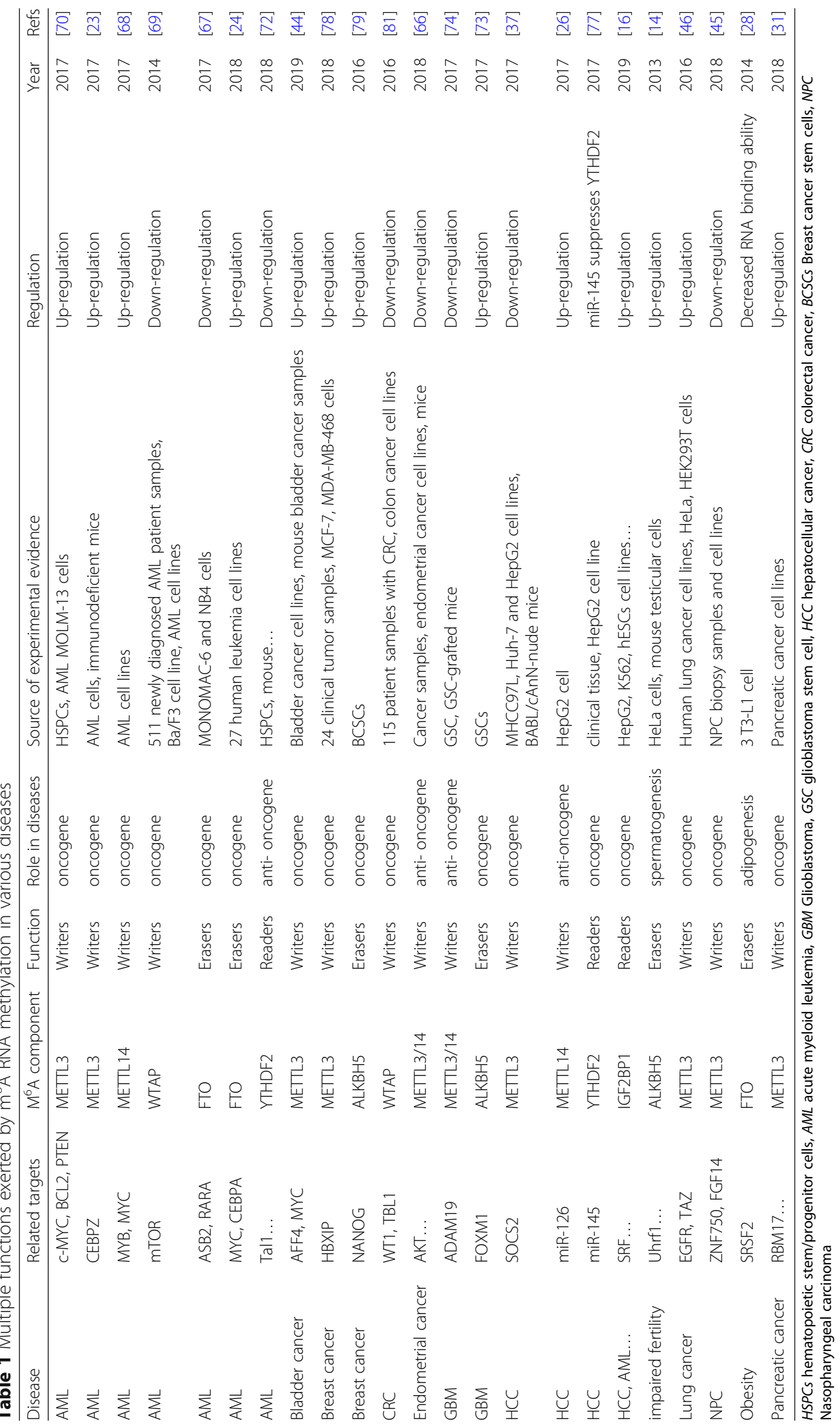




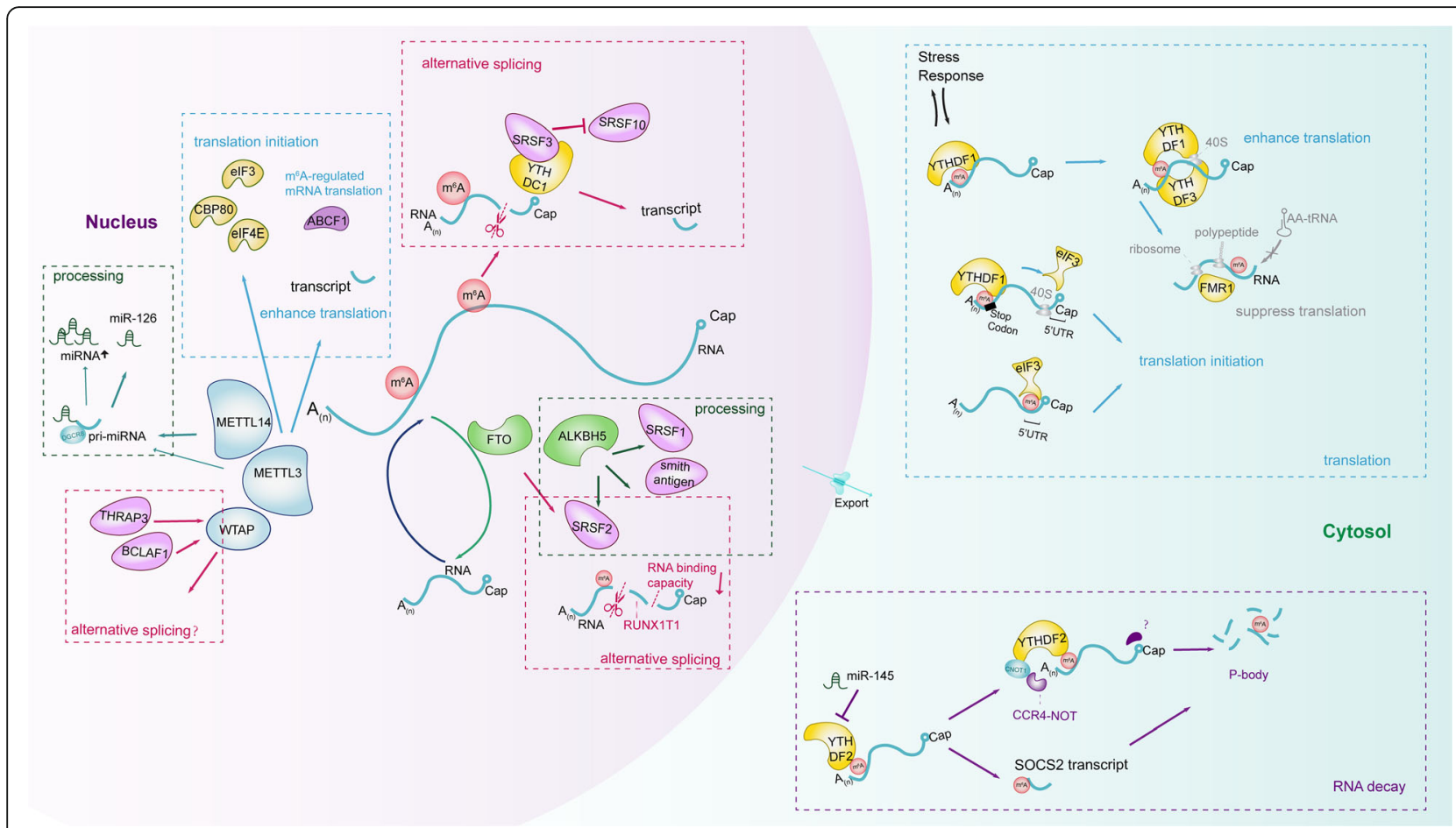

Fig. 2 Regulatory Functions of $\mathrm{m}^{6} \mathrm{~A}$ modification in RNA splicing, processing, translation and degradation. $\mathrm{M}^{6} \mathrm{~A}$ RNA modification is involved in regulating the life cycle of RNA including RNA splicing (regulated by WTAP, FTO, ALKBH5 and YTHDC1), RNA processing (regulated by METTL3/14 and ALKBH5), RNA translation (regulated by METTL3, YTHDF1/3, elF3 and FMR1) and RNA degradation (regulated by YTHDF2)

SOCS2 degradation also relies on $\mathrm{m}^{6} \mathrm{~A}$ 'reader' YTHDFs [37], which accelerate the decay of $\mathrm{m}^{6} \mathrm{~A}$-modified transcripts [38] or target mRNA [39]. Knockout of $\mathrm{m}^{6} \mathrm{~A}$ methyltransferase attenuates YTHDF2 specific binding with target mRNAs and increases their stability [40]. $\mathrm{M}^{6} \mathrm{~A}$ RNA methylation also controls $\mathrm{T}$ cell homeostasis by targeting the IL-7/STAT5/SOCS pathways [41] and decreases the stability of MYC/CEBPA transcripts [24].

\section{$M^{6} \mathrm{~A}$ modification in RNA translation}

$\mathrm{M}^{6} \mathrm{~A}$ modifications occur in mRNA and noncoding RNA (ncRNAs) to regulate gene expression in its $5^{\prime}$ or 3' UTR [7, 42]. METTL3 enhances mRNA translation [8], while depletion of METTL3 selectively inhibits mRNAs translation in 5'UTR [43] and reduces AFF4 and MYC translation in bladder cancer [44] but increase that of zinc finger protein 750 and fibroblast growth factor 14 in nasopharyngeal carcinoma [45].

$\mathrm{M}^{6} \mathrm{~A}$ modifications facilitate the initiated translation through interacting with the initiation factors eIF3, CBP80 and eIF4E in an RNA-independent manner [46]. Heat-shock-induced translation of heat-shock protein 70 (HSP70) alters the transcriptome-wide distribution of $\mathrm{m}^{6} \mathrm{~A}$ [7] and affects DNA repair [47]. ABCF1-sensitive transcripts largely overlaps with METTL3-modified mRNAs and are critical for $\mathrm{m}^{6} \mathrm{~A}$-regulated mRNA translation [43]. In addition, FMR1 binds to hundreds of
mRNAs to negatively regulate their translation [20]. YTHDF1 facilitates the translation of $\mathrm{m}^{6} \mathrm{~A}$-modified mRNAs in protein-synthesis and YTHDF3 acts in the initial stage of $\mathrm{m}^{6} \mathrm{~A}$-driven translation from circular RNAs (circRNAs) [38, 48, 49].

\section{$M^{6} A$ RNA modification in metabolic and developmental diseases}

The methyltransferases and demethylases of $\mathrm{m}^{6} \mathrm{~A}$ are associated with a variety of diseases, such as obesity [13, 50], type 2 diabetes mellitus $\left(\mathrm{T}_{2} \mathrm{DM}\right)$ [51], growth retardation, developmental delay, facial dysmorphism [52]. Besides, $\mathrm{m}^{6} \mathrm{~A}$ modification affects infertility [14], developmental arrest [22], neuronal disorder [53] and infectious diseases $[54,55]$.

\section{$M^{6} \mathrm{~A}$ modification in metabolic and infectious diseases}

$\mathrm{M}^{6} \mathrm{~A}$ modification is involved in metabolic abnormalities in patients with $\mathrm{T}_{2} \mathrm{DM}$ and obesity [56]. FTO regulates the energy homeostasis and dopaminergic pathway through $\mathrm{FTO}$-dependent $\mathrm{m}^{6} \mathrm{~A}$ demethylation $[50,51]$, and it is ubiquitous in adipose and muscle tissues, influencing RUNX1T1 splicing in adipogenesis $[28,30]$. METTL3/14 reduce the abundance of Hepatitis C virus replication, but FTO promotes its production through YTHDF proteins [54]. $\mathrm{M}^{6} \mathrm{~A}$ is also identified as a conserved modulatory symbol 
across Flaviviridae genomes, including dengue, Zika virus and West Nile virus [55].

\section{$M^{6} A$ modification in infertility}

Deficiency of demethylase ALKBH5 leads to the aberrant spermatogenesis and apoptosis with impaired fertility in testes and striking changes in DNA methyltransferase 1 (Dnmt1) and ubiquitin-like with PHD and RING finger domains 1 (Uhrf1) [14]. YTHDF2 is required for maternal transcriptome during oocyte maturation [57]. YTHDC1/2 determine the germline development in mouse [58], and YTHDC1 is essential for spermatogonia in males and oocyte maturation in females [27].

\section{$M^{6} \mathrm{~A}$ modification in nervous system development}

$\mathrm{M}^{6} \mathrm{~A}$ modification regulates the pace of cerebral cortex development [59] and $\mathrm{m}^{6} \mathrm{~A}$-regulated histone modifications enhances self-renewal of neural stem cells by METTL3/14 [60]. $\mathrm{M}^{6} \mathrm{~A}$ has dual effects on delaying tempo of corticogenesis by two distinct pathways: increased cell-cycle length and decreased mRNA decay [59]. $\mathrm{M}^{6} \mathrm{~A}$ depletion decreases the decay of radial glia cells associated with stem cell maintenance, neurogenesis and differentiation [61].

\section{$M^{6} \mathrm{~A}$ modification in inflammation and metabolism-related cancer}

Cacinogenesis is characterized by stepwise accumulation of genetic/epigenetic alterations of different protooncogenes and tumor-suppressor genes following other diseases including chronic inflammation and metabolic diseases. METTL3/14 and FTO influence Hepatitis C virus replication and production, and endogenous mediators of inflammatory responses (proinflammatory cytokines, reactive oxygen, et al) can promote genetic/ epigenetic alterations [62]. FTO affects RUNX1T1 splicing in adipogenesis $[28,30]$, and RUNX1T1 is essential for pancreas development [63]. Transcription factor forkhead box protein O1 (FOXO1) as another direct substrate of FTO, regulates gluconeogenesis in liver [64] and promotes the growth of pancreatic ductal adenocarcinoma [65].

\section{$M^{6} A$ RNA modification in human cancer}

Emerging evidence suggests that, $\mathrm{m}^{6} \mathrm{~A}$ modification is associated with the tumor proliferation, differentiation, tumorigenesis [46], proliferation [66], invasion [46] and metastasis [26] and functions as oncogenes or antioncogenes in malignant tumors (Table 1 and Fig. 3).

\section{Acute myeloid leukemia (AML)}

FTO is highly expressed in AML with t(11q23)/MLL rearrangements, t(15;17)/PML-RARA, FLT3-ITD and/or
NPM1 mutations and promotes leukemic cell transformation and tumorigenesis [67]. METTL3/14 are expressed in hematopoietic stem/progenitor cells (HSPCs) and AML cells with $t(11 \mathrm{q} 23), \mathrm{t}(15 ; 17)$, or $\mathrm{t}(8 ; 21)$, control the terminal myeloid differentiation of HSPCs and promote the survival and proliferation of AML [68]. WTAP acts in cell proliferation and arrests the differentiation of leukemia [69].

$\mathrm{M}^{6} \mathrm{~A}$ promotes the translation of $c-M Y C, B C L 2$ and PTEN in AML [70]. METTL14 acts an oncogenic role by regulating its targets $\mathrm{MYB} / \mathrm{MYC}$ through $\mathrm{m}^{6} \mathrm{~A}$ modification [68]. YTHDF2, responsible for the decay of $\mathrm{m}^{6} \mathrm{~A}$-modified mRNA transcripts [40], is also associated with MYC in leukemia [71]. Besides, YTHDF2 stabilizes Tal1 mRNAs and increases its expansion in AML [72].

Collectively, these studies corroborate the functional importance of $\mathrm{m}^{6} \mathrm{~A}$ modifications in leukemia, such as METTL3 [23, 70], METTL14 [68], FTO [24, 67] and YTHDF2 $[24,40]$ and they provide profound insights into development and maintenance of AML and selfrenewal of leukemia stem/initiation cells through the downstream MYC and Tal1 pathways.

\section{Glioblastoma (GBM)}

METTL3/14 inhibit GSC growth, self-renewal and tumorigenesis, but FTO and ALKBH5 indicate poor survival in GBM by regulating ADAM19 and transcription factor FOXM1 [73, 74]. LncRNA antisense to FOXM1 (FOXM1-AS) promotes the interaction of ALKBH5 with FOXM1 nascent transcripts in the tumorigenesis of GSCs [73].

\section{Lung cancer}

$\mathrm{M}^{6} \mathrm{~A}$ demethylase $\mathrm{FTO}$ is identified as a prognostic factor in lung squamous cell carcinoma (LUSC) and facilitates cell proliferation and invasion, but inhibits cell apoptosis by regulating MZF1 expression [75]. METTL3 acts as a oncogene in lung cancer by increasing EGFR and TAZ expression and promoting cell growth, survival and invasion [46]. METTL3-eIF3 caused mRNA circularization promotes the translation and oncogenesis of lung adenocarcinoma [46]. Besides, SUMOylation of METTL3 is of importance for the promotion of tumor growth at lysine residues $\mathrm{K}^{177}, \mathrm{~K}^{211}, \mathrm{~K}^{212}$ and $\mathrm{K}^{215}$ in non-small cell lung carcinoma (NSCLC) [76]. These studies provide insights into the critical roles of METTL3 and FTO in lung carcinoma.

\section{Hepatocellular carcinoma (HCC)}

METTL3 is related to a poor prognosis in HCC patients and promotes $\mathrm{HCC}$ cell proliferation, migration and colony formation by YTHDF2-dependent posttranscriptional silencing of SOCS2 [37]. But, METTL14 is an anti-metastatic factor and serves as a favorable factor in 


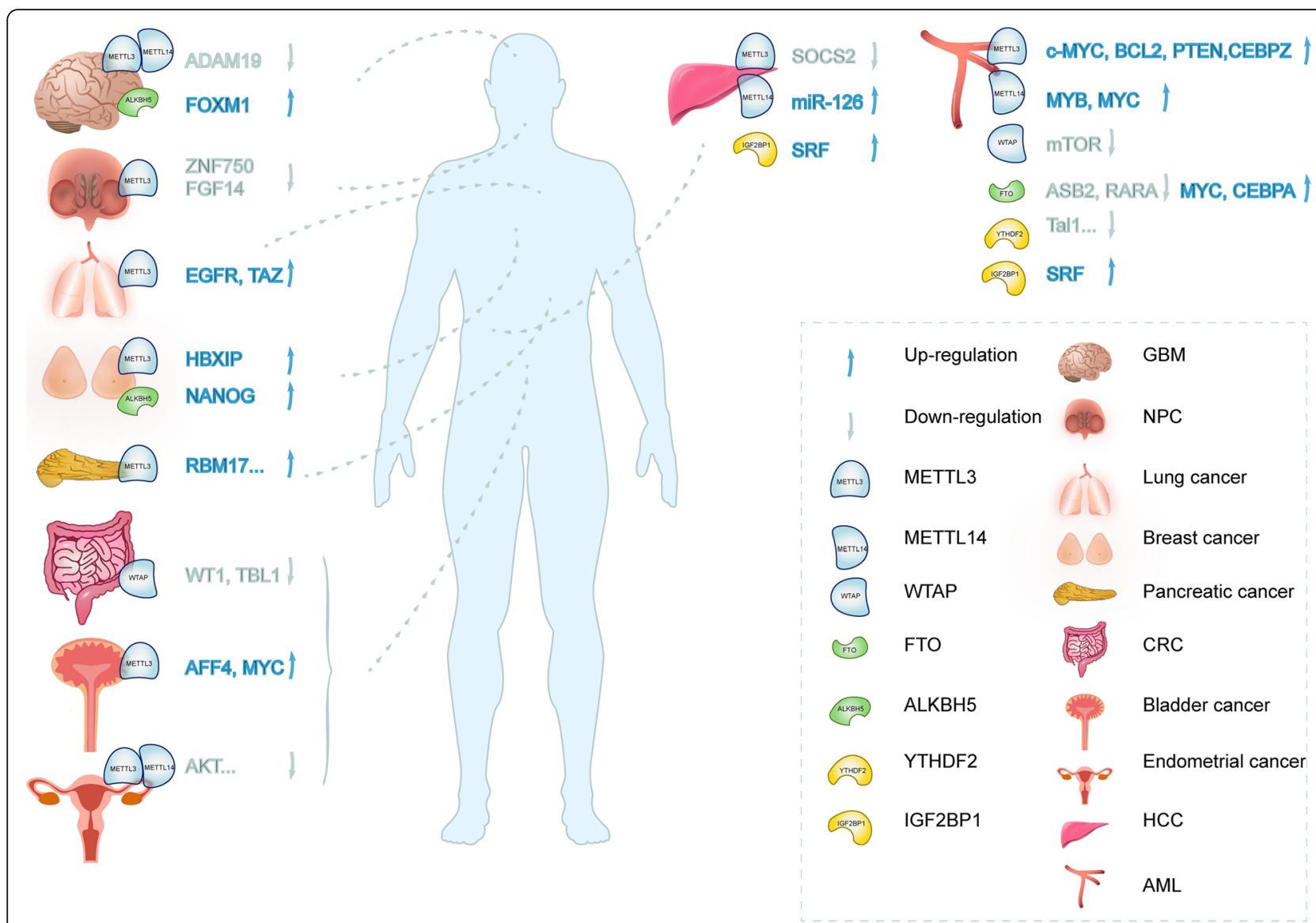

Fig. 3 The role of $m^{6} A$ RNA modification in human cancer. $M^{6} A$ RNA modification is associated with the tumorigenesis of multiple malignancies including AML, GBM, HCC, CRC, NPC, breast cancer, lung cancer, pancreatic cancer, bladder cancer and endometrial cancer

HCC by regulating $\mathrm{m}^{6} \mathrm{~A}$-dependent miRNA processing [26]. MiR-145 down-regulates YTHDF2 through targeting its mRNA 3' UTR [77]. In conclusion, METTL3 upregulation or METTL14 downregulation predicts poor prognosis in patients with $\mathrm{HCC}$ and contributes to $\mathrm{HCC}$ progression and metastasis [26, 37]. METTL3 suppresses SOCS2 expression in HCC via the $\mathrm{miR}-145 / \mathrm{m}^{6} \mathrm{~A} /$ YTHDF2 dependent axis [37, 77]. Thus, these studies suggest a new dimension of epigenetic alteration in liver carcinogenesis.

\section{Breast cancer and colorectal cancer (CRC)}

METTL3 is associated with the expression of mammalian hepatitis B X-interacting protein (HBXIP), displaying an aggressiveness in breast cancer. HBXIP-induced METTL3 promotes the proliferation of breast cancer via inhibiting tumor suppressor let-7 g [78]. Besides, ALKBH5 decreases the levels of $\mathrm{m}^{6} \mathrm{~A}$ in NANOG mRNA and enhances its stability, leading to an increase of NANOG mRNA and protein levels in breast cancer stem cells (BCSCs) [79]. Another $\mathrm{m}^{6} \mathrm{~A}$ eraser 'FTO' polymorphism has no association with the risk of CRC [80], but the $\mathrm{m}^{6} \mathrm{~A}$ 'writer'
WTAP is associated with carbonic anhydrase IV (CA4), which inhibits the proliferation and induces apoptosis and cycle arrest by repressing the Wnt signaling through targeting the WTAP-WT1-TBL1 axis [81].

\section{Brief summary of $m^{6} \mathrm{~A}$ modification-related carcinogenesis}

$\mathrm{M}^{6} \mathrm{~A}$ RNA modifications regulate RNA production/metabolism and take part in the carcinogenesis. On the one hand, $\mathrm{m}^{6} \mathrm{~A}$-modified genes usually act a oncogenic role in cancer, leading to alterations of mRNA translation and acceleration of tumor progression, and decreasing $\mathrm{m}^{6} \mathrm{~A}$ modification results in tumor development. On the other hand, given that SUMOylation of METTL3 represses its $\mathrm{m}^{6} \mathrm{~A}$ methyltransferase capacity and results in tumor growth of NSCLC, modification of $\mathrm{m}^{6} \mathrm{~A}$ methylase can determine the tumor development.

\section{$M^{6} \mathrm{~A}$ modification in cancer treatment}

$\mathrm{M}^{6} \mathrm{~A}$ modification indicates new directions for the treatment of various cancers. Regulators or inhibitors of $\mathrm{m}^{6} \mathrm{~A}$ modifications may provide the potential therapeutic strategies for cancers, such as MA2 in GBM [74], 
R-2HG/SPI1/FB23-2 in AML [24, 68, 82] and CA4 in CRC [81]. Meclofenamic acid (MA) as one of the selective FTO inhibitors is a non-steroidal anti-inflammatory drug by competing with FTO binding sites [83]. MA2, the ethyl ester derivative of $\mathrm{MA}$, increases $\mathrm{m}^{6} \mathrm{~A}$ modification, leading to the suppression of tumor progression $[74,83]$. The expression of ASB2 and RARA is increased in hematopoiesis and they act as key regulators of ATRAinduced differentiation of leukemia cells [84]. FTO enhances the leukemogenesis of AML by inhibition of the ASB2 and RARA expression [67]. FB23-2, as another inhibitor of $\mathrm{m}^{6} \mathrm{~A}$ demethylase FTO suppresses AML cell proliferation and promotes the cell differentiation and apoptosis [82].

ALKBH5 and FTO are $\alpha$-ketoglutarate $(\alpha-K G)$ dependent dioxygenases [85], which are competitively inhibited by D2-hydorxyglutarate (D2-HG) and elevated in isocitrate de-hydrogenases (IDH)-mutant cancers for transferring isocitrate to $\alpha$-KG [86]. R-2-hydroxyglutarate (R-2HG), an metabolite by mutating IDH1/2 enzyme, exhibits anti-leukemia effects through increasing $\mathrm{m}^{6} \mathrm{~A}$ levels in R-2HG-sensitive AML [24].

$\mathrm{S}$-adenosylmethionine (SAM) serves as a cofactor substrate in METTL3/14 complex and its product Sadenosylhomocysteine (SAH) inhibits the methyltransferases by competing with adenosylmethionine [87]. 3deazaadenosine (DAA) inhibits SAH hydrolase and interrupts insertion of $\mathrm{m}^{6} \mathrm{~A}$ into mRNA substrates [88] and its analogs suppress the replication of various viruses editing $\mathrm{m}^{6} \mathrm{~A}-\mathrm{mRNA}$ in cancers $[89,90]$.

METTL14 acts an oncogenic role by regulating MYB/ MYC axis through $\mathrm{m}^{6} \mathrm{~A}$ modification [68]. SPI1, a hematopoietic transcription factor, directly inhibits METTL14 expression in malignant hematopoietic cells [68] and may be a potential therapeutic target for AML. CA4 inhibits the tumorigenicity of CRC by suppressing the WTAP-WT1-TBL1 axis [81].

\section{Future prospect}

$\mathrm{M}^{6} \mathrm{~A}$ RNA modifications act by regulating RNA transcript, splicing, processing, translation and decay and participate in the tumorigenesis and metastasis of multiple malignancies. However, the underlying mechanisms of $\mathrm{m}^{6} \mathrm{~A}$ modifications in cancer should be further addressed.. Besides FMR1 and LRPPRC, the function of ALKBH family in $\mathrm{m}^{6} \mathrm{~A}$ RNA methylation is undetermined. METTL14 has different expression levels in various tumor tissues. Given a dual role of METTL14 either as a tumor suppressor [26] or an oncogene in cancer [68], its role in other cancers need be further elucidated. Though some inhibitors of $\mathrm{m}^{6} \mathrm{~A}$ methylation have shown promising effects on cancer development $[68,81]$, novel therapeutic strategies for $\mathrm{m}^{6} \mathrm{~A}$ RNA methylation should be further explored in the treatment of cancer.

\section{Abbreviations}

ALKBH5: Alkb homologue 5; AML: Acute myeloid leukemia; BCLAF1: BCL2associated transcription factor 1; BCSCS: Breast cancer stem cells; CA4: Carbonic anhydrase IV; CEBP: CCAAT-enhancer binding protein; circRNAs: Circular RNAs; CRC: Colorectal cancer; D2-HG: D2-hydorxyglutarate; DAA: 3-deazaadenosine; Dnmt1: DNA methyltransferase 1; elF: eukaryotic initiation factor; FGF14: Fibroblast growth factor 14; FOXM1-AS: Antisense to FOXM1; FOXO1: Forkhead box protein O1; FTO: Fat mass and obesityassociated protein; GSCs: Glioblastoma stem-like cells; HBXIP: Hepatitis B Xinteracting protein; HCC: Hepatocellular carcinoma; HNRNP: Heterogeneous nuclear ribonucleoprotein; HSP70: Heat-shock protein 70;

HSPCs: Hematopoietic stem/progenitor cells; IDH: Isocitrate de-hydrogenases; IGF2BP: IGF2 mRNA binding proteins; IncRNAs: Long non-coding RNAs; LUSC: Lung squamous cell carcinoma; $\mathrm{m}^{6} \mathrm{~A}$ : $\mathrm{N}^{6}$-methyladenosine; MA: Meclofenamic acid; METTL3: Methyltransferase-like 3; miRNAs: Micro RNAs; mRNAs: Messenger RNAs; ncRNAs: Noncoding RNAs; NSCLC: Non-small cell lung carcinoma; R-2HG: R-2-hydroxyglutarate; RUNX1T1: Runt-related transcription factor 1; SAH: S-adenosylhomocysteine; SAM: S-

adenosylmethionine; SOCS2: Suppressor of cytokine signaling 2; SRSF: serine/ arginine-rich splicing factor; $T_{2} D M$ : Type 2 diabetes mellitus; THRAP3: Thyroid hormone receptor-associated protein 3; Uhrf1: Ubiquitin-like with PHD and RING finger domains 1; UTR: Untranslated terminal region; WTAP: Wilms tumour 1-associated protein; YTHDF: YT521-B homology domain-containing protein family; ZNF750: Zinc finger protein 750; a-KG: A-ketoglutarate

\section{Acknowledgements}

Not applicable.

\section{Authors' contributions}

JZ and JSZ designed this study and XYC drafted the manuscript. JZ revised this manuscript. All authors read and approved the final manuscript.

\section{Funding}

Our work was supported by the grants from National Natural Science Foundation of China (No. 81873143; 81573747), and Shanghai Science and Technology Commission Western Medicine Guide project (No. 17411966500).

\section{Availability of data and materials}

All data generated or analyzed during this study are included in this published article and its additional files.

Ethics approval and consent to participate

Not applicable.

\section{Consent for publication}

All of the authors are aware of and agree to the content of the paper and their being listed as a co-author of the paper.

\section{Competing interests}

The authors declare that they have no competing interests.

Received: 18 March 2019 Accepted: 22 May 2019

Published online: 29 May 2019

References

1. Boccaletto P, Machnicka MA, Purta E, Piątkowski P, Bagiński B, Wirecki TK, et al. MODOMICS: a database of RNA modification pathways 2017 update. Nucleic Acids Res. 2018;46(Database issue):D303-7.

2. Desrosiers R, Friderici K, Rottman F. Identification of methylated nucleosides in messenger RNA from Novikoff hepatoma cells. Proc Natl Acad Sci U S A. 1974:71(10):3971-5.

3. Alarcón CR, Lee H, Goodarzi H, Halberg N, Tavazoie SF. N6-methyladenosine marks primary microRNAs for processing. Nature. 2015;519(7544):482-5.

4. Patil DP, Chen CK, Pickering BF, Chow A, Jackson C, Guttman M, et al. m6A RNA methylation promotes XIST-mediated transcriptional repression. Nature. 2016:537(7620):369-73.

5. Meyer KD, Saletore $Y$, Zumbo P, Elemento O, Mason CE, Jaffrey SR. Comprehensive analysis of mRNA methylation reveals enrichment in $3^{\prime}$ UTRs and near stop codons. Cell. 2012;149(7):1635-46. 
6. Ke S, Alemu EA, Mertens C, Gantman EC, Fak JJ, Mele A, et al. A majority of $\mathrm{m} 6 \mathrm{~A}$ residues are in the last exons, allowing the potential for 3' UTR regulation. Genes Dev. 2015;29(19):2037-53.

7. Meyer KD, Patil DP, Zhou J, Zinoviev A, Skabkin MA, Elemento O, et al. 5' UTR m6A promotes cap-independent translation. Cell. 2015;163(4):999-1010.

8. Schumann U, Shafik A, Preiss T. METTL3 gains R/W access to the Epitranscriptome. Mol Cell. 2016;62(3):323-4.

9. $\quad$ Liu J, Yue Y, Han D, Wang X, Fu Y, Zhang L, et al. A METTL3-METTL14 complex mediates mammalian nuclear RNA N6-adenosine methylation. Nat Chem Biol. 2014;10(2):93-5.

10. Ping $X L$, Sun BF, Wang $L$, Xiao W, Yang X, Wang W-J, et al. Mammalian WTAP is a regulatory subunit of the RNA N6-methyladenosine methyltransferase. Cell Res. 2014;24(2):177-89.

11. Meyer KD, Jaffrey SR. Rethinking $\mathrm{m}(6)$ a readers, writers, and erasers. Annu Rev Cell Dev Biol. 2017;33:319-42.

12. Schwartz S, Mumbach MR, Jovanovic M, Wang T, Maciag K, Bushkin GG, et al. Perturbation of m6A writers reveals two distinct classes of mRNA methylation at internal and 5' sites. Cell Rep. 2014;8(1):284-96.

13. Jia G, Fu Y, Zhao X, Dai Q, Zheng G, Yang Y, et al. N6-Methyladenosine in nuclear RNA is a major substrate of the obesity-associated FTO. Nat Chem Biol. 2011;7(12):885-7.

14. Zheng G, Dahl JA, Niu Y, Fedorcsak P, Huang C-M, Li CJ, et al. ALKBH5 is a mammalian RNA demethylase that impacts RNA metabolism and mouse fertility. Mol Cell. 2013;49(1):18-29.

15. Haussmann IU, Bodi Z, Sanchez-Moran E, Mongan NP, Archer N, Fray RG, et al. m6A potentiates $\mathrm{SxI}$ alternative pre-mRNA splicing for robust Drosophila sex determination. Nature. 2016;540(7632):301-4.

16. Müller S, Glaß M, Singh AK, Haase J, Bley N, Fuchs T, et al. IGF2BP1 promotes SRF-dependent transcription in cancer in a m6A- and miRNAdependent manner. Nucleic Acids Res. 2019;47(1):375-90.

17. Zhao BS, Roundtree IA, He C. Post-transcriptional gene regulation by mRNA modifications. Nat Rev Mol Cell Biol. 2017;18(1):31-42.

18. Xu C, Liu K, Ahmed H, Loppnau P, Schapira M, Min J. Structural basis for the discriminative recognition of N6-Methyladenosine RNA by the human YT521-B homology domain family of proteins. J Biol Chem. 2015:290(41):24902-13.

19. Arguello AE, DeLiberto AN, Kleiner RE. RNA chemical proteomics reveals the N6-Methyladenosine (m6A)-regulated protein-RNA Interactome. J Am Chem Soc. 2017;139(48):17249-52.

20. Edupuganti RR, Geiger S, Lindeboom RGH, Shi H, Hsu PJ, Lu Z, et al. N(6)methyladenosine $(\mathrm{m}(6) \mathrm{a})$ recruits and repels proteins to regulate mRNA homeostasis. Nat Struct Mol Biol. 2017;24(10):870-8.

21. Pendleton KE, Chen B, Liu K, Hunter OV, Xie Y, Tu BP, et al. The U6 snRNA m(6)a methyltransferase METTL16 regulates SAM Synthetase intron retention. Cell. 2017;169(5):824-835.e14.

22. Wei W, Ji X, Guo X, Ji S. Regulatory role of N6-Methyladenosine (m6A) methylation in RNA processing and human diseases. J Cell Biochem. 2017:2534-43

23. Barbieri I, Tzelepis K, Pandolfini L, Shi J, Millán-Zambrano G, Robson SC, et al Promoter-bound METTL3 maintains myeloid leukaemia by m6A-dependent translation control. Nature. 2017;552(7683):126-31.

24. Su R, Dong L, Li C, Nachtergaele S, Wunderlich M, Qing Y, et al. R-2HG exhibits anti-tumor activity by targeting $\mathrm{FTO} / \mathrm{m6A} / \mathrm{MYC} / \mathrm{CEBPA}$ signaling. Cell. 2018;172(1-2):90-105.e23.

25. Bartosovic M, Molares HC, Gregorova P, Hrossova D, Kudla G, Vanacova S. N6-methyladenosine demethylase FTO targets pre-mRNAs and regulates alternative splicing and $3^{\prime}$-end processing. Nucleic Acids Res. 2017:45(19):11356-70.

26. Ma JZ, Yang F, Zhou CC, Liu F, Yuan J-H, Wang F, et al. METTL14 suppresses the metastatic potential of hepatocellular carcinoma by modulating $N(6)$ -methyladenosine-dependent primary MicroRNA processing. Hepatol Baltim Md. 2017;65(2):529-43.

27. Kasowitz SD, Ma J, Anderson SJ, Leu NA, Xu Y, Gregory BD, et al. Nuclear m6A reader $Y T H D C 1$ regulates alternative polyadenylation and splicing during mouse oocyte development. PLoS Genet. 2018;14(5):e1007412.

28. Zhao $X$, Yang $Y$, Sun BF, Shi $Y$, Yang $X$, Xiao W, et al. FTO-dependent demethylation of N6-methyladenosine regulates mRNA splicing and is required for adipogenesis. Cell Res. 2014;24(12):1403-19.

29. Dominissini D, Moshitch-Moshkovitz S, Schwartz S, Salmon-Divon M, Ungar L, Osenberg S, et al. Topology of the human and mouse m6A RNA methylomes revealed by m6A-seq. Nature. 2012;485(7397):201-6.
30. Ben-Haim MS, Moshitch-Moshkovitz S, Rechavi G. FTO: linking m6A demethylation to adipogenesis. Cell Res. 2015;25(1):3-4.

31. Taketo K, Konno M, Asai A, Koseki J, Toratani M, Satoh T, et al. The epitranscriptome M6A writer METTL3 promotes chemo- and radioresistance in pancreatic cancer cells. Int J Oncol. 2018;52(2):621-9.

32. Horiuchi $K$, Kawamura $T$, Iwanari $H$, Ohashi R, Naito M, Kodama T, et al. Identification of Wilms' tumor 1-associating protein complex and its role in alternative splicing and the cell cycle. J Biol Chem. 2013;288(46):33292-302.

33. Rosa-Mercado NA, Withers JB, Steitz JA. Settling the m(6)a debate: methylation of mature mRNA is not dynamic but accelerates turnover. Genes Dev. 2017;31(10):957-8.

34. Ke S, Pandya-Jones A, Saito Y, Fak JJ, Vågbø CB, Geula S, et al. m6A mRNA modifications are deposited in nascent pre-mRNA and are not required for splicing but do specify cytoplasmic turnover. Genes Dev. 2017;31(10):990-1006.

35. Geula S, Moshitch-Moshkovitz S, Dominissini D, Mansour AA, Kol N, SalmonDivon $M$, et al. m6A mRNA methylation facilitates resolution of naïve pluripotency toward differentiation. Science. 2015;347(6225):1002-6.

36. Du H, Zhao Y, He J, Zhang Y, Xi H, Liu M, et al. YTHDF2 destabilizes m(6)Acontaining RNA through direct recruitment of the CCR4-NOT deadenylase complex. Nat Commun. 2016;7:12626.

37. Chen M, Wei L, Law CT, Tsang FH-C, Shen J, Cheng CL-H, et al. RNA N6methyladenosine methyltransferase-like 3 promotes liver cancer progression through YTHDF2-dependent posttranscriptional silencing of SOCS2. Hepatol Baltim Md. 2017;67(6):2254-70.

38. Shi H, Wang X, Lu Z, Zhao BS, Ma H, Hsu PJ, et al. YTHDF3 facilitates translation and decay of N(6)-methyladenosine-modified RNA. Cell Res. 2017:27(3):315-28.

39. Wang $X$, He C. Dynamic RNA modifications in posttranscriptional regulation. Mol Cell. 2014;56(1):5-12.

40. Wang $X$, Lu Z, Gomez A, Hon GC, Yue Y, Han D, et al. N6methyladenosine-dependent regulation of messenger RNA stability. Nature. 2014:505(7481):117-20.

41. Li HB, Tong J, Zhu S, Batista PJ, Duffy EE, Zhao J, et al. M(6)a mRNA methylation controls T cell homeostasis by targeting the IL-7/STAT5/SOCS pathways. Nature. 2017;548(7667):338-42.

42. Kennedy EM, Bogerd HP, Kornepati AVR, Kang D, Ghoshal D, Marshall JB, et al. Posttranscriptional m(6)a editing of HIV-1 mRNAs enhances viral gene expression. Cell Host Microbe. 2016;19(5):675-85.

43. Coots RA, Liu XM, Mao Y, Dong L, Zhou J, Wan J, et al. m6A facilitates elF4Findependent mRNA translation. Mol Cell. 2017;68(3):504-514.e7.

44. Cheng M, Sheng L, Gao Q, Xiong Q, Zhang H, Wu M, et al. The m6A methyltransferase METTL3 promotes bladder cancer progression via AFF4/ NF-kB/MYC signaling network. Oncogene. 2019;38(19):3667-80.

45. Zhang $P$, He Q, Lei Y, Li Y, Wen X, Hong M, et al. m6A-mediated ZNF750 repression facilitates nasopharyngeal carcinoma progression. Cell Death Dis. 2018;9(12):1169.

46. Lin S, Choe J, Du P, Triboulet R, Gregory RI. The m6A methyltransferase METTL3 promotes translation in human Cancer cells. Mol Cell. 2016;62(3):335-45

47. Xiang Y, Laurent B, Hsu CH, Nachtergaele S, Lu Z, Sheng W, et al. RNA m(6)a methylation regulates the ultraviolet-induced DNA damage response. Nature. 2017:543(7646):573-6.

48. Wang X, Zhao BS, Roundtree IA, Lu Z, Han D, Ma H, et al. N6methyladenosine modulates messenger RNA translation efficiency. Cell. 2015;161(6):1388-99.

49. Yang $Y$, Fan X, Mao M, Song X, Wu P, Zhang Y, et al. Extensive translation of circular RNAs driven by N(6)-methyladenosine. Cell Res. 2017;27(5):626-41.

50. Church C, Moir L, McMurray F, Girard C, Banks GT, Teboul L, et al. Overexpression of Fto leads to increased food intake and results in obesity. Nat Genet. 2010;42(12):1086-92.

51. Xiao S, Zeng X, Fan Y, Su Y, Ma Q, Zhu J, et al. Gene polymorphism association with type 2 diabetes and related Gene-gene and Geneenvironment interactions in a Uyghur population. Med Sci Monit Int Med J Exp Clin Res. 2016:22:474-87.

52. Boissel S, Reish O, Proulx K, Kawagoe-Takaki H, Sedgwick B, Yeo GSH, et al. Loss-of-function mutation in the dioxygenase-encoding FTO gene causes severe growth retardation and multiple malformations. Am J Hum Genet. 2009:85(1):106-11.

53. Maity A, Das B. N6-methyladenosine modification in mRNA: machinery, function and implications for health and diseases. FEBS J. 2016;283(9):1607-30. 
54. Gokhale NS, Mclntyre ABR, McFadden MJ, Roder AE, Kennedy EM, Gandara JA, et al. N6-Methyladenosine in Flaviviridae viral RNA genomes regulates infection. Cell Host Microbe. 2016;20(5):654-65.

55. Lichinchi G, Zhao BS, Wu Y, Lu Z, Qin Y, He C, et al. Dynamics of human and viral RNA methylation during Zika virus infection. Cell Host Microbe. 2016;20(5):666-73.

56. Gallagher EJ, LeRoith D. Obesity and diabetes: the increased risk of Cancer and Cancer-related mortality. Physiol Rev. 2015;95(3):727-48.

57. Ivanova I, Much C, Di Giacomo M, Azzi C, Morgan M, Moreira PN, et al. The RNA m(6)a reader YTHDF2 is essential for the post-transcriptional regulation of the maternal transcriptome and oocyte competence. Mol Cell. 2017;67(6).

58. Hsu PJ, Zhu Y, Ma H, Guo Y, Shi X, Liu Y, et al. Ythdc2 is an N(6)methyladenosine binding protein that regulates mammalian spermatogenesis. Cell Res. 2017;27(9):1115-27.

59. Boles NC, Temple S. Epimetronomics: m6A Marks the tempo of Corticogenesis. Neuron. 2017;96(4):718-20

60. Wang Y, Li Y, Yue M, Wang J, Kumar S, Wechsler-Reya RJ, et al. N6methyladenosine RNA modification regulates embryonic neural stem cell selfrenewal through histone modifications. Nat Neurosci. 2018;21(2):195-206.

61. Yoon K-J, Ringeling FR, Vissers C, Jacob F, Pokrass M, Jimenez-Cyrus D, et al. Temporal control of mammalian cortical neurogenesis by m6A methylation. Cell. 2017;171(4):877-889.e17.

62. Chiba T, Marusawa H, Ushijima T. Inflammation-associated cancer development in digestive organs: mechanisms and roles for genetic and epigenetic modulation. Gastroenterology. 2012;143(3):550-63.

63. Benitez CM, Qu K, Sugiyama T, Pauerstein PT, Liu Y, Tsai J, et al. An integrated cell purification and genomics strategy reveals multiple regulators of pancreas development. PLoS Genet. 2014;10(10):e1004645.

64. Peng S, Xiao W, Ju D, Sun B, Hou N, Liu Q, et al. Identification of entacapone as a chemical inhibitor of FTO mediating metabolic regulation through FOXO1. Sci Transl Med. 2019;(488):11.

65. Song W, Li Q, Wang L, Wang L. Modulation of FoxO1 expression by miR-2 to promote growth of pancreatic ductal adenocarcinoma. Cell Physiol Biochem Int J Exp Cell Physiol Biochem Pharmacol. 2015;35(1):184-90.

66. Liu J, Eckert MA, Harada BT, Liu S-M, Lu Z, Yu K, et al. m6A mRNA methylation regulates AKT activity to promote the proliferation and tumorigenicity of endometrial cancer. Nat Cell Biol. 2018;20(9):1074-83.

67. Li Z, Weng H, Su R, Weng X, Zuo Z, Li C, et al. FTO plays an oncogenic role in acute myeloid leukemia as a N6-Methyladenosine RNA demethylase. Cancer Cell. 2017;31(1):127-41.

68. Weng H, Huang H, Wu H, Qin X, Zhao BS, Dong L, et al. METTL14 inhibits hematopoietic stem/progenitor differentiation and promotes Leukemogenesis via mRNA m6A modification. Cell Stem Cell. 2017;22(2):191-205 e9.

69. Bansal H, Yihua Q, lyer S, Ganapathy S, Proia D, Penalva L, et al. WTAP is a novel oncogenic protein in acute myeloid leukemia. Leukemia. 2014;28(5):1171-4

70. Vu LP, Pickering BF, Cheng Y, Zaccara S, Nguyen D, Minuesa G, et al. The $\mathrm{N}(6)$-methyladenosine (m(6)a)-forming enzyme METTL3 controls myeloid differentiation of normal hematopoietic and leukemia cells. Nat Med. 2017:23(11):1369-76.

71. Wang H, Zuo H, Liu J, Wen F, Gao Y, Zhu X, et al. Loss of YTHDF2-mediated m6A-dependent mRNA clearance facilitates hematopoietic stem cell regeneration. Cell Res. 2018;28(10):1035-8.

72. Li Z, Qian P, Shao W, Shi H, He XC, Gogol M, et al. Suppression of m6A reader Ythdf2 promotes hematopoietic stem cell expansion. Cell Res. 2018;28(9):904-17.

73. Zhang S, Zhao BS, Zhou A, Lin K, Zheng S, Lu Z, et al. M(6)a demethylase ALKBH5 maintains Tumorigenicity of glioblastoma stem-like cells by sustaining FOXM1 expression and cell proliferation program. Cancer Cell. 2017;31(4):591-606.e6

74. Cui Q, Shi H, Ye P, Li L, Qu Q, Sun G, et al. M(6)a RNA methylation regulates the self-renewal and tumorigenesis of glioblastoma stem cells. Cell Rep. 2017:18(11):2622-34

75. Liu J, Ren D, Du Z, Wang H, Zhang H, Jin Y. m6A demethylase FTO facilitates tumor progression in lung squamous cell carcinoma by regulating MZF1 expression. Biochem Biophys Res Commun. 2018;502(4):456-64.

76. Du Y, Hou G, Zhang H, Dou J, He J, Guo Y, et al. SUMOylation of the m6ARNA methyltransferase METTL3 modulates its function. Nucleic Acids Res. 2018:46(10):5195-208.
77. Yang Z, Li J, Feng G, Gao S, Wang Y, Zhang S, et al. MicroRNA-145 modulates N(6)-Methyladenosine levels by targeting the 3'-untranslated mRNA region of the N(6)-Methyladenosine binding YTH domain family 2 protein. J Biol Chem. 2017:292(9):3614-23.

78. Cai X, Wang X, Cao C, Gao Y, Zhang S, Yang Z, et al. HBXIP-elevated methyltransferase METTL3 promotes the progression of breast cancer via inhibiting tumor suppressor let-7g. Cancer Lett. 2018;415:11-9.

79. Zhang C, Samanta D, Lu H, Bullen JW, Zhang H, Chen I, et al. Hypoxia induces the breast cancer stem cell phenotype by HIF-dependent and ALKBH5-mediated m6A-demethylation of NANOG mRNA. Proc Natl Acad Sci. 2016;113(14):E2047-56

80. Yang B, Thrift AP, Figueiredo JC, Jenkins MA, Schumacher FR, Conti DV, et al. Common variants in the obesity-associated genes FTO and MC4R are not associated with risk of colorectal cancer. Cancer Epidemiol. 2016;44:1-4.

81. Zhang J, Tsoi H, Li X, Wang H, Gao J, Wang K, et al. Carbonic anhydrase IV inhibits colon cancer development by inhibiting the Wnt signalling pathway through targeting the WTAP-WT1-TBL1 axis. Gut. 2016;65(9):1482-93.

82. Huang $Y$, Su R, Sheng Y, Dong L, Dong Z, Xu H, et al. Small-molecule targeting of oncogenic FTO demethylase in acute myeloid leukemia. Cancer Cell. 2019;35(4):677-91 e10.

83. Huang Y, Yan J, Li Q, Li J, Gong S, Zhou H, et al. Meclofenamic acid selectively inhibits FTO demethylation of m6A over ALKBH5. Nucleic Acids Res. 2015:43(1):373-84

84. Wang S, Sun C, Li J, Zhang E, Ma Z, Xu W, et al. Roles of RNA methylation by means of $\mathrm{N}(6)$-methyladenosine $(\mathrm{m}(6) \mathrm{a})$ in human cancers. Cancer Lett. 2017:408:112-20.

85. Fedeles Bl, Singh V, Delaney JC, Li D, Essigmann JM. The AlkB family of $\mathrm{Fe}(\mathrm{II}) /$ a-ketoglutarate-dependent dioxygenases: repairing nucleic acid alkylation damage and beyond. J Biol Chem. 2015;290(34):20734-42.

86. Lin AP, Abbas S, Kim S-W, Ortega M, Bouamar H, Escobedo Y, et al. D2HGDH regulates alpha-ketoglutarate levels and dioxygenase function by modulating IDH2. Nat Commun. 2015;6:7768.

87. Kloor D, Osswald H. S-Adenosylhomocysteine hydrolase as a target for intracellular adenosine action. Trends Pharmacol Sci. 2004;25(6):294-7.

88. Bader JP, Brown NR, Chiang PK, Cantoni GL. 3-Deazaadenosine, an inhibitor of adenosylhomocysteine hydrolase, inhibits reproduction of Rous sarcoma virus and transformation of chick embryo cells. Virology. 1978:89(2):494-505.

89. Mayers DL, Mikovits JA, Joshi B, Hewlett IK, Estrada JS, Wolfe AD, et al. Antihuman immunodeficiency virus 1 (HIV-1) activities of 3-deazaadenosine analogs: increased potency against 3'-azido-3'-deoxythymidine-resistant HIV-1 strains. Proc Natl Acad Sci U S A. 1995;92(1):215-9.

90. Gordon RK, Ginalski K, Rudnicki WR, Rychlewski L, Pankaskie MC, Bujnicki JM et al. Anti-HIV-1 activity of 3-deaza-adenosine analogs inhibition of Sadenosylhomocysteine hydrolase and nucleotide congeners. Eur J Biochem. 2003;270(17):3507-17.

\section{Publisher's Note}

Springer Nature remains neutral with regard to jurisdictional claims in published maps and institutional affiliations.
Ready to submit your research? Choose BMC and benefit from:

- fast, convenient online submission

- thorough peer review by experienced researchers in your field

- rapid publication on acceptance

- support for research data, including large and complex data types

- gold Open Access which fosters wider collaboration and increased citations

- maximum visibility for your research: over $100 \mathrm{M}$ website views per year

At BMC, research is always in progress.

Learn more biomedcentral.com/submissions 